\title{
Spatial variability in the physico-chemical properties of soils affected by animal wastes in Uyo, Akwa Ibom State of Nigeria
}

\author{
B. Ndukwu ${ }^{1 *}$, S. U. Onwudike ${ }^{1}$, M. C. Idigbor ${ }^{1}$, C. E. Ihejirika ${ }^{2}$ and K. S. Ewe ${ }^{1}$ \\ ${ }^{1}$ Department of Soil Science and Technology, Federal University of Technology Owerri, Imo State, Nigeria. \\ ${ }^{2}$ Department of Environmental Technology, Federal University of Technology Owerri, Imo State, Nigeria.
}

Accepted 14 January, 2013

\begin{abstract}
Variability in the physico-chemical properties of soils affected by animal wastes in Uyo, Akwa- Ibom State, Nigeria was investigated in this study. A free survey technique guided field sampling. Five profile pits were dug on five studied sites namely: Site severely affected with poultry manure, site moderately affected with poultry manure, site severely affected with swine manure, site moderately affected with swine manure, and control site (50 m away from the affected sites). Soil samples were collected at different depths and subjected to routine laboratory analysis. Data collected were analyzed statistically. Results of the investigation showed that variations existed among some physico-chemical properties of soils that are affected with animal wastes when compared to control. There were no variations in the silt and clay content of the affected soil. Among the affected soils, the $\mathrm{pH}$ and effective cation exchange capacity decreased down the profile. Results also showed that soils affected with poultry manure and swine manure had the highest exchangeable cations when compared to control. Appropriate measure should therefore be taken in the disposal of these wastes to avoid environmental hazards.
\end{abstract}

Key words: Variability, Epipedon, profile pits, environment, Akwa-Ibom State.

\section{INTRODUCTION}

With an increasing rate of human population worldwide, the rate of animal husbandry has been on the increase in order to meet up with food demand. Consequently, large quantities of animal wastes are produced which are deposited on soil as wastes. Research has shown that deposition of these animal wastes on soil increase soil organic matter and carbon fractions and enhances soil quality and productivity (Kingery et al., 1994). Application of animal wastes increase nutrient supplying capacity of the soil (Webster and Gouiding, 1989; Rochette and Gregorich, 1998), vegetative and reproductive growth of plants (Azam Shah et al., 2009; Suthar, 2009, Maftoun and Moshiri, 2008 and Sawyer et al., 2006), enhance moisture retention capacity and infiltration rate (Erikson et al., 1999), improve physical conditions of soil such as

\footnotetext{
*Corresponding author. E-mail: onwudikestanley@yahoo.com.
}

bulk density, aggregate stability and aeration (Yuksel and Orhan, 2004) as well as reduce the $\mathrm{pH}$ of an acid Ultisol (Bauer and Black, 1994) and crusting and runoff (Rochette and Gregorich, 1998).

Animal wastes are deposited on soil due to their nutrient value (Jackson and Bertsch, 2001; Garbarino et al., 2003), their effect on the environment has become an issue of interest (Mohammad et al., 2010). Studies have shown that long term deposition of animal waste on soil has resulted into ground and surface water pollution as a result of leaching and runoff of nutrients as well as accumulation of excessive soluble salts and the buildup of micronutrients (Mohammad et al., 2010; Zachary et al., 2008).

In Uyo, Akwa Ibom State Nigeria, large quantities of poultry manure and swine wastes abound in these areas and the effect of long term deposition on these materials on soil need to be investigated for proper environmental management and sustainability. Based on this premise 
therefore, this study was aimed at investigating the variability in the physico-chemical properties of soils affected by poultry manure and swine waste deposits.

\section{MATERIALS AND METHODS}

\section{Study area}

Uyo, Akwa Ibom State lies between latitude 4' 02' $\mathrm{N}$ and longitude 8' 21 'E. The area has a relative humidity of about 70 to $80 \%$. Soils were derived from coastal plain sand (Benin formation). It has a humid tropical climate with a mean annual rainfall of about 2000 to $2500 \mathrm{~mm}$ and an annual temperature of about 26 to $30^{\circ} \mathrm{C}$ (Uwah et al., 2011). The vegetation is that of the rainforest characterized by a variety of plants forms arranged in tiers. Soils have low nutrient content as a result of heavy rainfall that encourages leaching of basic cations. The socio-economic activity of people in this study site is growing of tropical crops like maize, cassava, plantain, okra, oil palm etc.

\section{Field study}

A free soil survey technique was used in this study. Five soil units were examined, namely soils severely affected with poultry manure, soils severely affected with swine waste, soils moderately affected with poultry manure $(50 \mathrm{~m}$ away from severely affected poultry manure), soils moderately affected with swine waste (50 m away from severely affected swine waste), and control which was $1.5 \mathrm{~km}$ away from soils affected with the animal manure. Five soil profile pits were sited in the entire studied area, representing a soil unit. The profile pits were dug and described according to the procedures of FAO (1990) with depths of 1 to 20,20 to 40,40 to 60 and 60 to $80 \mathrm{~cm}$, thereby making a total of 20 soil samples. These samples were air dried and sieved using $2 \mathrm{~mm}$ mesh sieve and subjected to routine laboratory analysis.

\section{Laboratory analysis}

Particle size distribution was determined by hydrometer method (Gee and Or, 2002) using Sodium hexametaphosphate (Calcon) as dispersant. Soil $\mathrm{pH}$ was determined electronically in a 1: 2.5 soil: solution ratio (Hendershot et al., 1993). Bulk density was estimated using core sampler calculated by mass of oven dried soil divided by volume of core sampler (Foth, 1984). Soil moisture content was determined gravimetrically. Total Nitrogen was determined by Kjeldal digestion method using concentrated $\mathrm{H}_{2} \mathrm{SO}_{4}$ and Sodium Copper Sulphate as catalyst mixture (Bremner and Yeomans, 1988). Organic carbon was determined by wet oxidation method (Nelson and Sommers, 1982). Exchangeable bases were determined using $1 \mathrm{~N}$ ammonium acetate solution according to Jackson (1964). Exchangeable Calcium (Ca) and Magnesium (Mg) were determined using Ethylene Diamine tetracetic acid (EDTA) while exchangeable Sodium (Na) and Potassium (K) were determined flame photometrically. Exchangeable acidity $\left(\mathrm{Al}^{3+}\right.$ and $\mathrm{H}^{+}$) was determined according to Mclean (1982). Available phosphorus was determined by extraction with Bray II solution and determined calorimetrically on spectrophotometer according to Nelson and Sommers (1982). Effective Cation Exchange Capacity (ECEC) was computed by the summation of all exchangeable bases and exchangeable acidity while percentage base saturation was calculated by dividing total exchangeable bases with effective cation exchangeable capacity and the quotient multiplied by 100 . Soil data were subjected to mean descriptive statistics and correlation and coefficient of variation analysis were used to ascertain the variability according to Aweto (1982).

\section{RESULTS AND DISCUSSION}

Some selected physical properties of the soil are shown in Table 1. The soils are texturally more of sandy loam. There was more clay fraction in soils moderately affected with poultry manure than soils severely affected with either poultry or swine waste. Dominance of sand fractions in the studied site could be attributed to the parent material (coastal plain sand) from which the soils are formed as well as humid rainfall characteristic that promote leaching of silt and clay fractions down the macro-porous soils. Since most of the secondary minerals are domicile in clay fractions of the soil, moderate application of swine waste on soil with resultant increase in clays could boost soil fertility because of an increase in ion exchange reactions.

There were variations in the bulk density of the soil among the treatments. There was a lower bulk density on soils affected with animal wastes when compared to the control. This could be attributed to the role of organic wastes in reducing the bulk density of the soil and this was in concord with Brady and Weil (1999) who noted that application of animal wastes either for plant nutrient supply or for disposal purposes reduces soil bulk density because of their ability in forming soil aggregates.

Soil moisture retention also varied among the soils but the highest moisture content was recorded on soils severely affected with either poultry manure or swine waste and the moisture content increases down the soil. This could be attributed to the mulching effect of these materials as well as improved structure and macroporosity. This observation was in agreement with Aluko and Oyedele (2005) who noted that application of organic wastes increases the water retention capacity of soil. However, the mobility and retentivity of water may depend on the activation energy of the clays which could be influenced by the type of basic cations (Logsdon and Laird, 2004). This observation could be vital in the management of surface and ground water pollution since severe application of animal waste could cause ground water pollution (Mohammad et al., 2010).

The chemical properties of the soils are shown in Table 2. In each of the studied area, soil pH decreases down the profile with control location having the lowest results due to low accumulation of organic matter and soils severally affected with poultry manure recorded the highest values. The acidity of the soils could be attributed to the acid parent material from where the soils are formed and the mineralization of organic wastes which releases organic acids (fulvic and humic acid) which are leached down the profile hence, decreasing the $\mathrm{pH}$ value. Soils affected with poultry manure and swine waste recorded higher exchangeable Calcium and Magnesium than the control. This could be attributed to the improvement in soil $\mathrm{pH}$, since $\mathrm{pH}$ range of 5.5 to 7.0 
Table 1. Some physical properties of the studied soils.

\begin{tabular}{|c|c|c|c|c|c|c|c|}
\hline \multirow{2}{*}{ Depth } & \multirow{2}{*}{$\begin{array}{l}\text { Sand } \\
(\mathrm{g} / \mathrm{kg})\end{array}$} & Silt (g/kg) & Clay $(\mathrm{g} / \mathrm{kg})$ & $\mathrm{BD}(\mathrm{g} / \mathrm{cm} 3)$ & MC (\%) & \multirow{2}{*}{$\begin{array}{c}\text { Total porosity } \\
\text { (\%) }\end{array}$} & \multirow{2}{*}{ Textural class } \\
\hline & & \multicolumn{4}{|c|}{ Profile Pit 1 (Severally affected with poultry manure) } & & \\
\hline $0-20$ & 785.2 & 152 & 62.8 & 1.20 & 1.80 & 55 & Sandy loam \\
\hline $20-40$ & 775.2 & 132 & 92.8 & 1.26 & 2.00 & 52 & Sandy loam \\
\hline $40-60$ & 735.2 & 192 & 82.8 & 1.43 & 2.07 & 46 & Loamy sand \\
\hline $60-80$ & 755.7 & 172 & 72.7 & 1.38 & 2.42 & 48 & Sandy sand \\
\hline \multirow[t]{2}{*}{ Mean } & 762.7 & 162 & 77.8 & 1.32 & 2.07 & 50 & \\
\hline & & \multicolumn{4}{|c|}{ Profile Pit 2 (moderately affected with poultry manure) } & & \\
\hline $0-20$ & 825.2 & 132 & 42.7 & 1.24 & 1.58 & 53 & Sandy loam \\
\hline $20-40$ & 765.2 & 192 & 42.8 & 1.30 & 1.55 & 51 & Sandy loam \\
\hline $40-60$ & 715.2 & 252 & 32.7 & 1.19 & 1.89 & 55 & Sandy clay loam \\
\hline $60-80$ & 672.2 & 292 & 32.8 & 1.13 & 1.49 & 57 & Sandy clay \\
\hline \multirow[t]{2}{*}{ Mean } & 694.5 & 217 & 37.8 & 1.22 & 1.63 & 54 & \\
\hline & & \multicolumn{4}{|c|}{ Profile Pit 3 (Severely affected with Swine manure) } & & \\
\hline $0-20$ & 735.2 & 142 & 102.8 & 1.08 & 2.57 & 59 & Sandy loam \\
\hline $20-40$ & 805.2 & 162 & 32.7 & 1.07 & 2.27 & 60 & Sandy loam \\
\hline $40-60$ & 725.2 & 252 & 22.8 & 1.26 & 1.44 & 52 & Sandy clay loam \\
\hline $60-80$ & 705.2 & 262 & 32.8 & 1.13 & 2.17 & 57 & Sandy clay \\
\hline \multirow[t]{2}{*}{ Mean } & 742.7 & 204.5 & 47.4 & 1.14 & 2.11 & 57 & \\
\hline & & \multicolumn{4}{|c|}{ Profile Pit 4 (Moderately affected with Swine manure) } & & \\
\hline $0-20$ & 775.2 & 152 & 72.8 & 1.14 & 1.31 & 57 & Sandy loam \\
\hline $20-40$ & 755.2 & 182 & 62.6 & 1.27 & 1.15 & 52 & Sandy loam \\
\hline $40-60$ & 712.2 & 242 & 42.9 & 1.19 & 1.17 & 55 & Sandy clay loam \\
\hline $60-80$ & 735.2 & 202 & 62.8 & 1.25 & 1.39 & 53 & Sandy clay loam \\
\hline \multirow[t]{2}{*}{ Mean } & 744.5 & 194.5 & 60.3 & 1.21 & 1.26 & 54 & \\
\hline & & \multicolumn{4}{|c|}{ Profile Pit 5 (Control) } & & \\
\hline $0-20$ & 685.2 & 252 & 62.8 & 1.92 & 1.26 & 28 & Loamy sand \\
\hline $20-40$ & 655.2 & 272 & 72.8 & 1.81 & 1.27 & 32 & Sandy clay loam \\
\hline $40-60$ & 635.2 & 292 & 72.7 & 1.76 & 1.22 & 34 & Sandy clay loam \\
\hline $60-80$ & 645.2 & 302 & 52.9 & 1.87 & 1.15 & 29 & Sandy clay loam \\
\hline Mean & 655.2 & 279.5 & 65.3 & 1.84 & 1.23 & 31 & \\
\hline
\end{tabular}

$\mathrm{BD}$, Bulk density; MC, moisture content.

favors the availability of exchangeable cations. The observation was in concord with Khaleel et al. (1981) who reported that high soil acidity lowers the availability of Calcium, Sodium, Phosphorus and Potassium due to the production of nitrate.

There was no effect on exchangeable Sodium in all the pedons indicating unavailability of soluble salts in the area or in the animal wastes used in this study. High precipitation common in the area could lead to dissolution and leaching of any soluble salt that might have been introduced into the soil through the animal foods. In all the pedons, Calcium dominated other cations. This was in agreement with Braver et al. (1978). Calcium saturated soils have lower activation energy, hence its water retention is high and therefore its hydration energy is low. Increase in the concentration of Calcium therefore increases aggregate stability of soil particles since Calcium act as a binding agent holding soil particles and organic polymers together (Baver et al., 1978). Application of animal waste therefore increases soil aggregation.

The effective cation exchange capacity (ECEC) decreases down the profile in all the soil. This could be due to decrease in organic matter content down the profile since increase in organic matter increases the cation exchange capacity (Onwudike, 2010). The same trend was observed in percentage base saturation. There is a positive correlation between organic matter and base saturation as also reported by Bell and Moody (1998).

There was moderate organic carbon in the epipedon (mainly A- horizon) in all the soil. This was due to organic matter availability but higher organic carbon was found in soils affected with animal wastes than in control. Low total Nitrogen and organic carbon recorded in this study could be as a result of high mineralization of organic manure associated with tropical environment which leads to leaching of available organic matter due to high precipitation. Application of poultry manure and swine wastes increased the availability of Phosphorus which was 
Table 2. Soil chemical properties of the study site.

\begin{tabular}{|c|c|c|c|c|c|c|c|c|c|c|c|c|c|}
\hline $\begin{array}{l}\text { Depth } \\
\text { (cm) }\end{array}$ & $\mathrm{pH}\left(\mathrm{H}_{2} \mathbf{0}\right)$ & $\mathrm{pH}(\mathrm{KCl})$ & $\begin{array}{l}\mathrm{Al}^{2+} / \mathrm{H}^{-} \\
(\mathrm{Cmol})\end{array}$ & $\begin{array}{c}\mathrm{Ca}^{2+} \\
\text { (Cmol) }\end{array}$ & $\begin{array}{c}\mathrm{Mg}^{2+} \\
\text { (Cmol) }\end{array}$ & $\begin{array}{c}\mathrm{Na}^{+} \\
(\mathrm{Cmol})\end{array}$ & $\begin{array}{c}\mathrm{K}^{+} \\
(\mathrm{Cmol})\end{array}$ & $\begin{array}{c}\text { TEB } \\
\text { (Cmol) }\end{array}$ & $\begin{array}{c}\text { ECEC } \\
\text { (Cmol) }\end{array}$ & $\begin{array}{l}\text { BS } \\
(\%)\end{array}$ & $\begin{array}{l}\text { OC } \\
(\%)\end{array}$ & $\begin{array}{l}\mathrm{TN} \\
(\%)\end{array}$ & $\begin{array}{l}\text { Av. P } \\
\text { (ppm) }\end{array}$ \\
\hline & & & & & & & \multicolumn{7}{|c|}{ Profile Pit 1} \\
\hline $0-20$ & 6.43 & 5.60 & 0.95 & 1.56 & 0.80 & 0.01 & 0.03 & 2.40 & 3.35 & 72 & 1.7 & .04 & 17.5 \\
\hline $20-40$ & 5.55 & 4.88 & 1.10 & 1.50 & 0.86 & 0.02 & 0.03 & 2.41 & 3.51 & 69 & 1.6 & .03 & 14.7 \\
\hline $40-60$ & 5.25 & 4.35 & 1.30 & 0.90 & 0.56 & 0.01 & 0.01 & 1.50 & 2.80 & 54 & 1.2 & .02 & 13.6 \\
\hline $60-80$ & 4.80 & 4.01 & 0.90 & 0.68 & 0.21 & 0.02 & 0.01 & 0.92 & 1.82 & 51 & 1.2 & .03 & 12.9 \\
\hline Mean & 5.51 & 4.71 & 1.60 & 1.16 & 0.61 & 0.02 & 0.02 & 1.81 & 2.87 & 62 & 1.4 & .03 & 14.7 \\
\hline \multicolumn{14}{|c|}{ Profile Pit 2} \\
\hline $0-20$ & 5.21 & 4.14 & 0.65 & 1.34 & 1.10 & $\operatorname{Tr}$ & $\operatorname{Tr}$ & 1.10 & 1.75 & 63 & 1.6 & .03 & 15.4 \\
\hline $20-40$ & 4.52 & 3.64 & 0.55 & 0.90 & 0.76 & 0.01 & $\operatorname{Tr}$ & 0.77 & 1.27 & 61 & 1.2 & .03 & 13.3 \\
\hline $40-60$ & 4.35 & 3.55 & 0.45 & 0.70 & 0.56 & 0.01 & $\operatorname{Tr}$ & 0.57 & 1.02 & 56 & 1.2 & .02 & 9.8 \\
\hline $60-80$ & 4.48 & 3.58 & 0.50 & 0.62 & 0.20 & 0.01 & $\operatorname{Tr}$ & 0.29 & 1.71 & 30 & 1.1 & .01 & 8.4 \\
\hline Mean & 4.64 & 3.73 & 0.54 & 0.89 & 0.66 & & & 0.66 & 1.44 & 53 & 1.3 & .02 & 11.7 \\
\hline \multicolumn{14}{|c|}{ Profile Pit 3} \\
\hline $0-20$ & 5.42 & 3.77 & 0.75 & 1.42 & 1.36 & 0.02 & 0.03 & 2.83 & 3.58 & 79 & 1.8 & .04 & 22.4 \\
\hline $20-40$ & 4.36 & 3.29 & 0.50 & 1.38 & 0.90 & 0.02 & 0.04 & 2.34 & 2.84 & 82 & 1.3 & .04 & 16.1 \\
\hline $40-60$ & 4.24 & 3.20 & 1.30 & 1.26 & 0.44 & 0.01 & 0.03 & 1.74 & 3.04 & 57 & 1.2 & .02 & 14.0 \\
\hline $60-80$ & 4.30 & 3.22 & 0.70 & 0.96 & 0.34 & 0.01 & 0.02 & 1.33 & 2.03 & 66 & 1.2 & .02 & 13.3 \\
\hline Mean & 4.58 & 3.37 & 0.81 & 1.26 & 0.76 & 0.02 & 0.03 & 2.06 & 2.87 & 72 & 1.4 & .03 & 16.5 \\
\hline \multicolumn{14}{|c|}{ Profile Pit 4} \\
\hline $0-20$ & 5.86 & 4.82 & 0.55 & 1.24 & 0.70 & $\mathrm{Tr}$ & 0.02 & 0.72 & 1.27 & 57 & 1.7 & .03 & 16.8 \\
\hline $20-40$ & 4.79 & 3.94 & 0.96 & 0.86 & 0.36 & 0.01 & 0.01 & 0.74 & 1.70 & 44 & 1.4 & .03 & 13.8 \\
\hline $40-60$ & 4.47 & 3.63 & 1.00 & 0.38 & 0.38 & 0.01 & 0.01 & 0.48 & 1.78 & 44 & 1.3 & .02 & 9.1 \\
\hline $60-80$ & 4.12 & 3.23 & 0.72 & 0.18 & 0.18 & 0.01 & 0.01 & 0.38 & 1.10 & 35 & 1.1 & .01 & 6.9 \\
\hline Mean & 4.81 & 3.91 & 0.59 & 0.54 & 0.41 & & 0.01 & 0.66 & 1.46 & 45 & 1.4 & .02 & 11.7 \\
\hline \multicolumn{14}{|c|}{ Profile Pit5 } \\
\hline $0-20$ & 4.79 & 3.75 & 1.20 & 0.56 & 0.18 & 0.01 & $\operatorname{Tr}$ & 0.19 & 1.39 & 14 & 1.5 & .03 & 14.0 \\
\hline $20-40$ & 4.34 & 3.57 & 1.35 & 0.50 & 0.32 & 0.01 & $\operatorname{Tr}$ & 0.33 & 1.68 & 20 & 1.4 & .02 & 11.2 \\
\hline $40-60$ & 4.10 & 3.23 & 0.80 & 0.66 & 0.12 & $\mathrm{Tr}$ & $\mathrm{Tr}$ & 0.12 & 0.92 & 13 & 1.2 & .01 & 9.1 \\
\hline $60-80$ & 3.80 & 3.95 & 1.15 & 0.60 & 0.20 & 0.01 & $\mathrm{Tr}$ & 0.22 & 1.67 & 13 & 1.2 & .01 & 6.7 \\
\hline Mean & 4.26 & 3.39 & 1.20 & 0.58 & 0.21 & & & 0.22 & 1.42 & 15 & 1.3 & .02 & 10.3 \\
\hline
\end{tabular}

TN, Total Nitrogen; OC, organic carbon; Av.P, available Phosphorus; TEB, Total exchangeable bases; ECEC, effective cation exchange capacity; BS, base saturation; Tr, trace.

found higher than in control. This could be attributed to favourable $\mathrm{pH}$ range and total $\mathrm{N}$ which complements each other. Some of the relationships existing among soil physicochemical properties are shown in Tables 3 and 4.

Results showed that positive correlation existed
$(P=0.01)$ among the selected physico-chemical properties in the studied locations (Table 3). No significant difference existed between moisture, 
Table 3. Correlation coefficient among physico-chemical properties of soil in the study site.

\begin{tabular}{|c|c|c|c|c|c|c|c|c|c|c|c|c|c|}
\hline Parameter & $\begin{array}{l}\text { Sand } \\
(\mathrm{g} / \mathrm{kg})\end{array}$ & $\begin{array}{c}\text { Clay } \\
(\mathrm{g} / \mathrm{kg})\end{array}$ & $\begin{array}{c}\text { Silt } \\
(\mathrm{g} / \mathrm{kg})\end{array}$ & $\begin{array}{c}\text { BD } \\
\left(\mathrm{g} / \mathrm{cm}^{3}\right)\end{array}$ & $\begin{array}{l}\text { MC } \\
(\%)\end{array}$ & $\begin{array}{l}\mathrm{TN} \\
(\%)\end{array}$ & $\begin{array}{c}\mathrm{pH} \\
\left(\mathrm{H}_{2} \mathrm{O}\right)\end{array}$ & $\underset{(\mathrm{KCl})}{\mathrm{pH}}$ & $\begin{array}{l}\text { OC } \\
(\%)\end{array}$ & $\begin{array}{l}\text { Av. P } \\
\text { (ppm) }\end{array}$ & $\begin{array}{c}\text { TEA } \\
\text { (ppm) }\end{array}$ & $\begin{array}{c}\text { TEB } \\
(\mathrm{Cmol} / \mathrm{kg})\end{array}$ & $\begin{array}{c}\text { ECEC } \\
(\mathrm{Cmol} / \mathrm{kg})\end{array}$ \\
\hline Clay $(\mathrm{g} / \mathrm{kg})$ & $0.95^{\star *}$ & & & & & & & & & & & & \\
\hline Silt $(g / k g)$ & $0.04^{\mathrm{NS}}$ & $0.43^{N S}$ & & & & & & & & & & & \\
\hline $\mathrm{BD}\left(\mathrm{g} / \mathrm{cm}^{3}\right)$ & $0.68^{\star}$ & $0.53^{*}$ & $0.04^{\mathrm{NS}}$ & & & & & & & & & & \\
\hline $\mathrm{MC}(\%)$ & $0.27^{\mathrm{NS}}$ & $0.19^{\mathrm{NS}}$ & $0.21^{\mathrm{NS}}$ & $0.58^{*}$ & & & & & & & & & \\
\hline $\mathrm{TN}(\%)$ & $0.70^{*}$ & $0.87^{\star \star}$ & $0.45^{*}$ & $0.33^{N S}$ & $0.14^{\mathrm{NS}}$ & & & & & & & & \\
\hline $\mathrm{pH}\left(\mathrm{H}_{2} \mathrm{O}\right)$ & $0.58^{\star *}$ & $0.76^{\star *}$ & $0.52^{*}$ & $0.37^{\mathrm{NS}}$ & $0.19^{N S}$ & $0.86^{\star *}$ & & & & & & & \\
\hline $\mathrm{pH}(\mathrm{KCl})$ & $0.59^{* *}$ & $0.77^{\star *}$ & $0.46^{\mathrm{NS}}$ & $0.39^{N S}$ & $0.21^{\mathrm{NS}}$ & $0.88^{\star *}$ & $0.98^{\star *}$ & & & & & & \\
\hline $\mathrm{OC}(\%)$ & $0.43^{N S}$ & $0.67^{\star *}$ & $0.54^{*}$ & $0.04^{N S}$ & $0.19^{N S}$ & $0.81^{* *}$ & $0.82^{* *}$ & $0.83^{* *}$ & & & & & \\
\hline Av. $P(p p m)$ & $0.62^{\star *}$ & $0.76^{\star \star}$ & $0.36^{\mathrm{NS}}$ & $0.22^{N S}$ & $0.09^{N S}$ & $0.93^{\star *}$ & $0.76^{\star *}$ & $0.74^{\star *}$ & $0.76^{* *}$ & & & & \\
\hline TEA $(\mathrm{Cmol} / \mathrm{kg})$ & $0.41^{\mathrm{NS}}$ & $0.22^{\mathrm{NS}}$ & $0.29^{\mathrm{NS}}$ & $0.24^{\mathrm{NS}}$ & $0.12^{\mathrm{NS}}$ & $0.14^{\mathrm{NS}}$ & $0.49^{* *}$ & 0.01 * & 0.01 * & $0.12^{N S}$ & & & \\
\hline TEB (Cmol/kg & $0.72^{\star *}$ & $0.73^{\star *}$ & $0.23^{\mathrm{NS}}$ & $0.29^{N S}$ & $0.07^{\mathrm{NS}}$ & $0.79^{* *}$ & $0.80^{* *}$ & $0.61^{* *}$ & $0.66^{* *}$ & $0.81^{* *}$ & $0.26^{\mathrm{NS}}$ & & \\
\hline ECEC $(\mathrm{Cmol} / \mathrm{kg}$ & $0.29^{N S}$ & $0.48^{*}$ & $052^{*}$ & $0.0^{\mathrm{NS}}$ & $0.06^{N S}$ & $0.91^{\star *}$ & $0.52^{\star *}$ & $0.53^{* *}$ & $0.64^{* *}$ & $0.58^{* *}$ & $0.53^{* *}$ & $0.68^{* *}$ & \\
\hline BS (\%) & $0.77^{\star *}$ & $0.65^{\star *}$ & $0.05^{\mathrm{NS}}$ & $0.39^{N S}$ & $0.02^{N S}$ & $0.63^{\star *}$ & $0.47^{* *}$ & $0.47^{\star *}$ & $0.48^{* *}$ & $0.64^{* *}$ & $0.62^{* *}$ & $0.86^{\star *}$ & $0.26^{\mathrm{NS}}$ \\
\hline
\end{tabular}

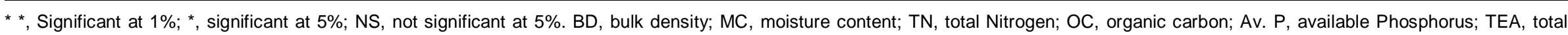
exchangeable acidity; TEB, total exchangeable bases; ECEC, effective cation exchange capacity; BS, base saturation.

Table 4. Variability in the physico-chemical properties of the studied soils.

\begin{tabular}{|c|c|c|c|c|c|c|c|c|c|c|c|c|c|c|}
\hline $\begin{array}{l}\text { Depth } \\
\text { (cm) }\end{array}$ & $\begin{array}{l}\text { Sand } \\
(g / k g)\end{array}$ & $\begin{array}{c}\text { Silt } \\
(\mathrm{g} / \mathrm{kg})\end{array}$ & $\begin{array}{c}\text { Clay } \\
(\mathrm{g} / \mathrm{kg})\end{array}$ & $\begin{array}{l}\text { BD } \\
(\%)\end{array}$ & $\begin{array}{l}\text { MC } \\
(\%) \\
\end{array}$ & $\begin{array}{c}\mathrm{pH} \\
\left(\mathrm{H}_{2} \mathrm{O}\right)\end{array}$ & $\begin{array}{c}\mathrm{Al}^{3+} \mathrm{H}^{+} \\
(\mathrm{Cmol} / \mathrm{kg})\end{array}$ & $\begin{array}{c}\text { TEB } \\
(\mathrm{Cmol} / \mathrm{kg}) \\
\end{array}$ & $\begin{array}{l}\text { ECE ECEC } \\
(\mathrm{Cmol} / \mathrm{kg})\end{array}$ & $\begin{array}{c}\mathrm{pH} \\
(\mathrm{KCl})\end{array}$ & $\begin{array}{l}\text { BS } \\
(\%)\end{array}$ & $\begin{array}{l}\text { OC } \\
(\%)\end{array}$ & $\begin{array}{l}\mathrm{TN} \\
(\%) \\
\end{array}$ & $\begin{array}{l}\text { Av. P } \\
\text { (ppm) }\end{array}$ \\
\hline \multicolumn{15}{|c|}{ Profile Pit 1 (Severally affected with poultry manure) } \\
\hline Mean & 762.7 & 162 & 77.8 & 1.3 & 1.7 & 5.5 & 5.7 & 1.1 & 1.8 & 2.9 & 61.1 & 1.4 & 0.03 & 14.7 \\
\hline$\% \mathrm{CV}$ & 2.62 & 16.6 & 15.9 & 8.04 & 24.7 & 12.5 & 14.7 & 16.9 & 46.7 & 22.8 & 33.8 & 19.9 & 22.8 & 13.9 \\
\hline Rank & $\mathrm{L}$ & M & M & L & M & L & $\mathrm{L}$ & $\mathrm{M}$ & $\mathrm{H}$ & M & $\mathrm{H}$ & M & M & $\mathrm{L}$ \\
\hline \multicolumn{15}{|c|}{ Profile Pit 2 (Moderately affected with poultry manure) } \\
\hline Mean & 694.5 & 217 & 37.8 & 1.2 & 1.6 & 4.6 & 3.7 & 0.5 & 0.7 & 1.4 & 52.2 & 1.3 & 0.02 & 11.7 \\
\hline$\% \mathrm{CV}$ & 8.7 & 15.3 & 32.3 & 6 & 11 & 8.3 & 7.5 & 15.8 & 56.2 & 37 & 29.5 & 10.7 & 36.6 & 27.3 \\
\hline Rank & $\mathrm{L}$ & M & $\mathrm{H}$ & $\mathrm{L}$ & $\mathrm{L}$ & $\mathrm{L}$ & $\mathrm{L}$ & M & $\mathrm{H}$ & $\mathrm{H}$ & $\mathrm{H}$ & $\mathrm{L}$ & $\mathrm{H}$ & $\mathrm{H}$ \\
\hline \multicolumn{15}{|c|}{ Profile Pit 3 (Severely affected with Swine manure) } \\
\hline Mean & 742.7 & 207.5 & 47.4 & 1.1 & 2.1 & 4.6 & 3.9 & 0.8 & 2.1 & 2.9 & 71.1 & 1.4 & 0.03 & 16.5 \\
\hline$\% C V$ & 5.8 & 17.3 & 30 & 8 & 22.7 & 12.3 & 10.8 & 42.2 & 59.4 & 31.7 & 42.2 & 19.7 & 36.2 & 25.2 \\
\hline Rank & $\mathrm{L}$ & $\mathrm{M}$ & $\mathrm{H}$ & $\mathrm{L}$ & $\mathrm{M}$ & $\mathrm{L}$ & L & $\mathrm{H}$ & $\mathrm{H}$ & $\mathrm{H}$ & $\mathrm{H}$ & M & $\mathrm{H}$ & $\mathrm{M}$ \\
\hline \multicolumn{15}{|c|}{ Profile Pit 4 (Moderately affected with Swine manure) } \\
\hline Mean & 744.5 & 194.5 & 60.3 & 1.2 & 2.3 & 4.8 & 3.9 & 0.6 & 0.7 & 1.5 & 44.7 & 1.4 & 0.02 & 11.7 \\
\hline$\% \mathrm{CV}$ & 3.4 & 20.9 & 19.4 & 4.9 & 7.7 & 15.6 & 18.9 & 24.4 & 50.9 & 21.4 & 36.7 & 17.3 & 33.7 & 38.6 \\
\hline
\end{tabular}


Table 4. Contd.

\begin{tabular}{|c|c|c|c|c|c|c|c|c|c|c|c|c|c|c|}
\hline Rank & $\mathrm{L}$ & $M$ & $M$ & $\mathrm{~L}$ & $\mathrm{~L}$ & $M$ & $\mathrm{M}$ & $M$ & $\mathrm{H}$ & $M$ & $\mathrm{H}$ & $M$ & $\mathrm{H}$ & $\mathrm{H}$ \\
\hline \multicolumn{15}{|c|}{ Profile pit 5 (Control) } \\
\hline Mean & 655.2 & 279.5 & 65.3 & 1.84 & 1.23 & 4.3 & 3.4 & 1.2 & 0.2 & 1.4 & 14.9 & 1.3 & 0.02 & 10.3 \\
\hline$\% C V$ & 3.3 & 14.7 & 16.9 & 8.3 & 27.4 & 9.3 & 11.9 & 23.8 & 40.6 & 25.2 & 21.4 & 10.8 & 48.9 & 32.1 \\
\hline Rank & $\mathrm{L}$ & $\mathrm{L}$ & $\mathrm{M}$ & $\mathrm{L}$ & $\mathrm{H}$ & $\mathrm{L}$ & $\mathrm{L}$ & M & $\mathrm{H}$ & $\mathrm{H}$ & $\mathrm{M}$ & $\mathrm{L}$ & $\mathrm{H}$ & $\mathrm{H}$ \\
\hline
\end{tabular}

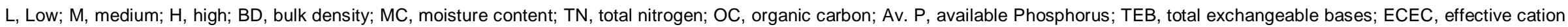
exchange capacity; BS, base saturation; CV, coefficient of variation.

bulk density content and selected soil properties except between moisture content and bulk density. In all the studied locations, variability existed among the physico-chemical properties except sand, bulk density and total exchangeable bases (Table 4). Variability existing among these soil properties may be attributed to land use system, (Esu et al., 1991) and differences in the mineral composition of these organic wastes.

\section{CONCLUSION AND RECOMMENDATION}

Hitherto, dumping of animal wastes on soils has been one of the effective measures of improving soil fertility and productivity. However, long term dumping of these wastes has started posing an environmental and management problem such as surface and ground water pollution eutrophication, as well as leaching of mineral salts. Results of this investigation have shown that some degree of variability exists among soil properties that are affected with poultry manure and swine waste when compared to unaffected soils. Some of these variations are prominent down the profile due to leaching of mineral elements. From the findings of this study, it is recommended that long term deposition of organic wastes should be discouraged. A sustainable approve such as utilizing them as organic fertilizers should be adopted than dumping them as means of disposal.

\section{REFERENCES}

Aluko OB, Oyedele DJ (2005). Influence of organic waste incorporation on changes in selected soil physical properties. J. Appl. Sci. 5(2):357-362.

Aweto AO (1982). Variability of upper slope soils developed under sand stone in Southeastern Nigeria. J. Geol. 25:2737.

Azam Shah SS. Mohmood S, Mohammad W, Shafi M, Nawaz $H$ (2009). N. uptake and yield of wheat as influenced by integrated use of organic and mineral nitrogen. Int. J. Plant Prod. 3 (3): $45-56$

Bauer A, Black AL (1994). Quantification of the effect of soil organic matter content on soil productivity. Am. J. Soil. Sci. 5:185-193

Braver I.D, Gardner WH, Gardner WR (1978). Soil physics. In: John and Sons, fourth Ed., New York. p. 498.

Bremner JM, Yeomans JC (1988). Laboratory Techniques in J.R. Wilson (ed.). Advances in Nitrogen cycling in Agricultural ecosystem. C.A.B Int. England.

Bell MJ, Moody PW (1998). The role of active fraction of soil organic matter in physical and chemical fertility of ferrosols. Aust. J. soil Res. 36:309-819.

Brady NC, Weil RR (1999). The nature and properties of soils. $12^{\text {th }}$. Ed., Prentice Hall Inc., New Jersey. p. 860

Erikson G, Coale F, Bellero G (1999). soil nutrient dynamics and maize production in organic waste amended soil. Soll Sci. Soc. Am. J. 15:85- 92.

Esu IE, Odunze AC, Morberg, JP (1991). Physico-chemical and mineralogical properties of the soils in Talata-Mafara area of Sokoto. Samaru J. Agric. Res. 8:41 -56. FAO (Food and Agricultural Organisation). 1990. Guidelines for soil profile description. 3th Ed, FAO, Rome. p. 70.
Foth HD (1984). Fundamentals of soil Science. $7^{\text {th }}$ Ed., John Wiley and Sons, New York. p 435.

Garbarino JR, Bednar AJ. Rutherford DW, Beyer RS, Wershaw R.L (2003). Environmental fate of roxarsone in poultry litter. I. Degradation of roxarsone during composting. Environ. Sci. Technol. 37:1509-1514.

Gee GW,.Or D (2002). Particle Size analysis. In: Dane, J. H and G.C.Topps (ed.). Methods of soil analysis, Part 4, Physical Methods. Soil Science Society of America, Book Series, No. 5, asa and SSSA Madison WI. pp.255-293.

Hendershot WH, Lalande H, Duquette M (1993). Soil reaction and exchangeable acidity. In : Carter, M.R (Ed.). Soil Sampling and Methods of Soil Analysis. Canadian Society of Soil Science, Lewis Publishers, London. pp.141- 145.

Jackson ML (1964). Soil Chemical Analysis: Prentice Hall Englewood Cliffs, N.J: pp.86-92.

Jackson BP, Bertsch PM (2001). Determination of arsenic speciation in poultry wastes by IC-ICP-MS. Environ. Sci. Technol.35: 4868-4873.

Khaleel R, Reddy KR, Overcash MR (1981). Changes in soil physical and chemical properties due to application of animal manure. J. Environ. Quality. 326:126-178.

Kingery WL, Wood CW, Delaney DP, Williams JC, Mullins GI (1994). Impact of long-term land application of broiler litter on environmentally related soil properties. J. Environ. Quality. 23:139-147.

Logsdon S, Laird D (2004). Cation and water content on dopole rotation activation energy of smectites. Soil Sci. Soc. Am. J. 64:54-61.

Maftoun M, Moshiri F (2008). Growth, mineral nutrition and selected soil properties of lowland rice, as affected by soil application of organic wastes and phosphorus. J. Agric. Sci. Technol. 10:481-492.

Mclean EV (1982). Aluminum. In: Page , A.I., R. H. Miller and D.R. Keeney (ed.). Method of soil analysis, Part 2. $2^{\text {nd }}$ ed., Agron,Monpgr p 9, ASA and SSSA Madison, WI. pp. 978998. 
Mohammad RM, Serda A, Kobra K (2010). Using animal manure for improving soil chemical properties under different leaching conditions. Res. J. Soil Water Manage. (1):34-37. Nelson DW, Sommers EL (1982). Total carbon, organic carbon and organic matter. In: Page, A.L. (ED.). Methods of soil analysis. Part 2. $2^{\text {nd }} \mathrm{Ed}$. Madison, WI, ASA and SSSA. pp 149-157.

Onwudike SU (2010). Effectiveness of cow dung and mineral fertilizer on soil properties, nutrient uptake and yield of sweet potato (Ipomoea batatas) in Southeastern Nigeria. Asian. J. Agric. Res. 4 (3):148-154.

Rochette P, Gregorian EG (1998). Dynamics of soil microbial biomass carbon, soluble organic carbon and $\mathrm{CO}_{2}$ evolution after three years of manure application. Can. J. Soil Sci. 78:283-290.

Sawyer J, Helmers M, . Mallarino A, Lamkey K, Baker J (2006). Environmental Protection Commission-Alternative Considerations Regarding Liquid Swine Manure Application Rates to Soybean. Iowa State University, Ames, IA.

Suthar S (2009). Impact of vermicompost and composted farmyard manure on growth and yield of garlic (Allium sativum L.) field crop. Int. J. Plant Prod. 3:27-38.
Uwah DF, Udoh AU, Iwo GA (2011). Effect of Organic and Mineral Fertilizers on Growth and Yield of Cocoyam (Colocasia Esculenta (L.) SCHOTT). Int. J. Agric. Sci. 3(1):33-38.

Webster CP, Goulding KWT (1989). Influence of soil Carbon content on denitrification from fallow land during autumn. J. Sci. Food Agric. 49:131-142.

Yukse, BX, Orhan Y (2004). Effect of swine manure on some chemical characteristics of clay soil. J. Agric. 3(1):43- 45.

Zachary NS, Ermson ZN,, A.T. Ivenus AT, Chandra KR (2008). Tillage cropping systems and nitrogen fertilizer source; effects on soil carbon sequestration and fraction. In: J. Environ. Quality.37:880-885 